\title{
Incidence and Susceptibility Pattern of Methicillin Resistant Coagulase- Negative Staphylococci Isolated From a Tertiary Care Hospital of Pakistan
}

\author{
Muhammad Usman Shah ${ }^{1, *}$; Muhammad Farhan Akram ${ }^{1}$; Javaid Usman ${ }^{1}$; Fatima Kaleem ${ }^{1}$ \\ ${ }^{1}$ Department of Microbiology, Army Medical College, National University of Sciences and Technology, Islamabad, Pakistan \\ ${ }^{*}$ Corresponding author: Muhammad Usman Shah, Army Medical College, National University of Sciences and Technology, House 516, Street 5, sector C4, Phase 5, Hayatabad, Pesha- \\ war, Khyber Pakhtunkhwa, Islamabad, Pakistan. Tel:+92-3469187704, E-mail: usman_shah44@msn.com \\ Received: October 14, 2012; Revised: January 12, 2013; Accepted: January 21, 2013
}

\begin{abstract}
Background: Methicillin resistant coagulase-negative Staphylococci are resistant organisms causing infections associated with high morbidity and mortality. Methicillin resistant Staphylococcus epidermidis (MRSE), is especially important with respect to admitted patients with indwelling catheters and other installed invasive devices where these organisms are known to be found. As a result, such lifesaving measures may prove fatal from subsequent infection and sepsis by these pathogens. Therefore, to limit such conditions in patients, the spread of MRSE and related organisms in the hospitals have to be effectively controlled.

Objectives: This study was carried out to determine the frequency of methicillin resistant organisms among all isolated coagulase negative Staphylococci (CoNS) and to find effective antibiotics against these microorganisms.

Patients and Methods: All samples sent to the lab were routinely processed according to standard microbiological procedures and the cultures yielding growth of CoNS were selected for the study. All samples containing CoNS collected over a 2 year-period, were included irrespective of patients' age and gender. The antibiogram of the organisms was recorded according to CLSI guidelines and the ratio of methicillin resistant organisms determined.

Results: From a total of 299 isolated coagulase negative Staphylococci(CoNS), 40.1\% were methicillin resistant. A high proportion of these organisms (more than 50\%) were resistant to cephalosporins, aminoglycosides and quinolones while only a small number were found to show resistance to linezolid, minocycline, chloramphenicol and rifampicin. There were no resistant organisms against vancomycin. Conclusions: A considerable amount of methicillin resistant organisms found among CoNS in our region. The above stated antibiotics would prove effective in limiting these infections. Clinicians should keep these facts in mind while treating their patients.
\end{abstract}

Keywords: Antibiotics; Coagulase Negative Staphylococci; Methicillin Resistance

\section{Background}

Staphylococcus epidermidis is the main organism among the coagulase negative Staphylococci (CoNS). It is a part of the normal flora of the skin but may act as a pathogen causing fatal infections which may have a significant incidence especially in the immune compromised patients (1). Likewise, it is also one of the most frequent organism causing post-operative surgical site and graft infections (2) as well as intravascular catheter and prostheses infection (3). The last mentioned infection is particularly significant as the increasing adoption of invasive procedures, prosthetic implants and percutaneous devices are used which are resulted in a high probability of subsequent infections. S. epidermidis and CoNS continue to be the major causes of sepsis (4) and meningitis in neonates and elderly hospitalized patients admitted for atopic dermatitis (2).

The increasing number of resistant organisms causes difficulties to treat life threatening infections. This is a consequence of mass use of antibiotics (1) resulting in emergence of resistant genes giving rise to organisms like methicillin resistant S. epidermidis (MRSE). Therefore, with the reduced effectiveness of previous antibiotics and a rise of nosocomial infections, these resistant organisms resulted in increased morbidity and mortality rate of admitted patients into our hospitals (5). The resistance may be increased several folds with the production of enveloping biofilms. Such organisms are especially found in indwelling catheters and other instruments. These biofilmassociated catheter infections, responsible for recurrent CoNS infections in hospitalized premature neonates, are difficult to treat because of intrinsic resistance of biofilms to antibiotics (4).

Currently, vancomycin is considered as the drug for eradicating such resistant organisms. However, cases of coagulase negative Staphylococci resistant to vancomycin are now appearing, as a majority that has developed the above mentioned biofilms (6). This makes these organisms extremely resistant to this antibiotic, resulting in therapeutic failure of the drug. Therefore, other drugs that have been recently introduced for such organisms, including linezolid, along with a few traditional ones such as minocycline, rifampicin and fusidic acid may

Implication for health policy/practice/research/medical education:

The implication of this study was to effectively control the CoNS associated resistant infections in our hospitals in order to reduce the morbidity and mortality and overall financial burden on our health care system.

Copyright @ 2014,Ahvaz Jundishapur University of Medical Sciences; Published by Kowsar Corp. This is an open-access article distributed under the terms of the Creative Commons Attribution License, which permits unrestricted use, distribution, and reproduction in any medium, provided the original work is properly cited. 
need to be used which may prove to be more effective in treating such infections. Other means to overcome this resistance is the modification of the dosage regimens (e.g. using high-dose therapy), inhibiting the resistance mechanism (e.g. beta-lactamase inhibitors) or using an agent from a different class (7).

\section{Objectives}

Our study was aimed to determine the incidence of methicillin resistance among all of the coagulase negative Staphylococci isolated and to determine the effectiveness of various antibiotics to find out a suitable, yet cost effective treatment against these resistant organisms.

\section{Patients and Methods}

A descriptive, cross sectional study was carried out in the Microbiology department of Army Medical College, National University of Sciences and Technology, to determine the frequency and antibiogram of the methicillin resistance of all the CoNS isolated in the lab. This study was performed during 2 years, from January 2008 to January 2010. An informed consent form and institutional review board approval was received from the Army Medical College/ Military Hospital Pakistan review board. Informed consent was also taken from all the patients involved in the study. The studied samples were including blood, pus, urine, intravenous catheter tips, double lumen tip samples, catheter tip samples, umbilical swabs and wound swabs collected from both hospital admitted and outdoor patients.

Each sample was cultured onto blood agar plates and incubated at $37^{\circ} \mathrm{C}$ for 24 hours. The characteristic isolates were aseptically isolated and identified as Staphylococcus by standard methods, including colonial morphology, Gram staining and catalase test. The isolates with coagulase and DNAase test positive results were considered as $S$. aureus and those with negative results were considered as CoNS. Final identification was done by API Staph kit and results were interpreted using API manufacturer manual instruction. Oxacillin $(1 \mu \mathrm{g})$ and cefoxitin $(30 \mu \mathrm{g})$ disks were used to assess the susceptibility of the isolates to methicillin.

The isolates were considered as methicillin resistant if the zone of inhibition was $<25 \mathrm{~mm}$ for cefoxitin. Antibiotic susceptibility of the isolates as methicillin resistant was tested by Kirby-Bauer disc diffusion as recommended by the Clinical and Laboratory Standards Institute (1997). The following antibiotic disks (Oxoid-UK) were used: cephalaxin $(30 \mu \mathrm{g})$, cephradine $(30 \mu \mathrm{g})$, ciprofloxacin (5 $\mu \mathrm{g})$, quinopristin/dalfopristin (15 $\mu \mathrm{g})$, gentamicin (30 g), erythromycin (15 g), levofloxacin $(5 \mu \mathrm{g})$, tetracycline (30 $\mathrm{g})$, rifampicin (5 g), teicoplanin (30 g), minocycline (30 $\mu \mathrm{g})$, chloramphenicol $(30 \mu \mathrm{g})$, linezolid $(30 \mu \mathrm{g})$ and vancomycin $(30 \mu \mathrm{g})$. The isolates were inoculated on Mueller Hinton Agar (Oxoid-UK) containing 5\% $\mathrm{NaCl}$ and incubated at $35^{\circ} \mathrm{C}$ for 24 hours.

All isolates, irrespective of the age and sex of the pa- tient, were included in the study and the cultures yielding growth of coagulase-negative Staphylococci were selected for the study and their antibiogram recorded according to CLSI guidelines. Data was entered in and analyzed using "Microsoft Excel 2007". The selected strains in this study had the potential of being clinically significant on the basis of source and quantity and infective agent or both.

\section{Results}

Out of 299 coagulase negative Staphylococci isolates, 120 (40.1\%) were found to be methicillin resistant which isolated from pus, urine, blood and other miscellaneous samples (Figure 1). The most effective antibiotics were vancomycin, linezolid, chloramphenicol, minocycline, and rifampicin. The efficacy of the first two was 100\% followed by minocycline with only $4 \%$ effectiveness on isolated resistant organisms. Chloramphenicol and rifampicin was moderately effective with $21.9 \%$ and $31.8 \%$ resistance. Antibiotics that proved ineffective included fuscidic acid (44\%), quinolones (87.3\%) and aminoglycosides (71.6\%) (Figure 2).

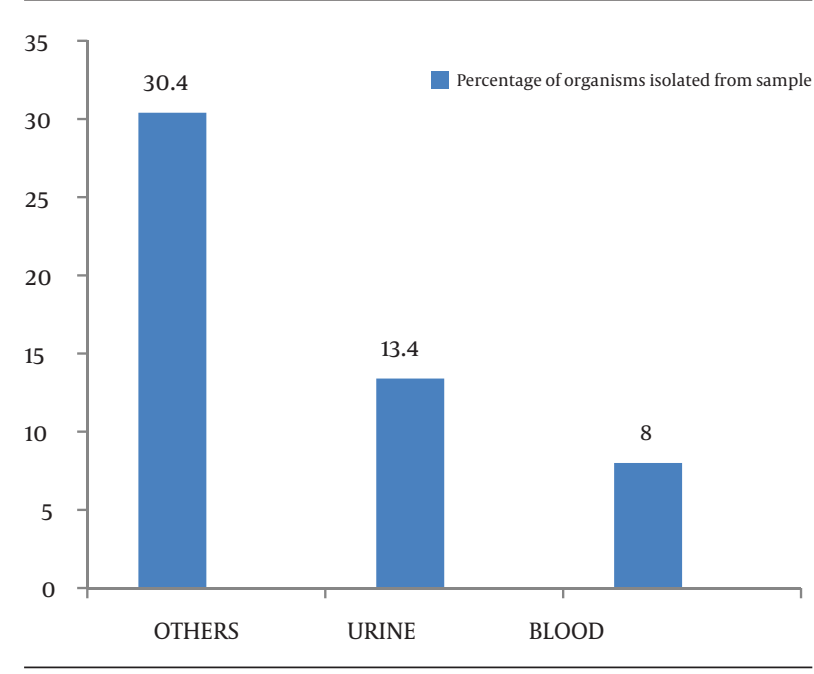

Figure 1. Frequency of Organisms Isolated From Various Samples

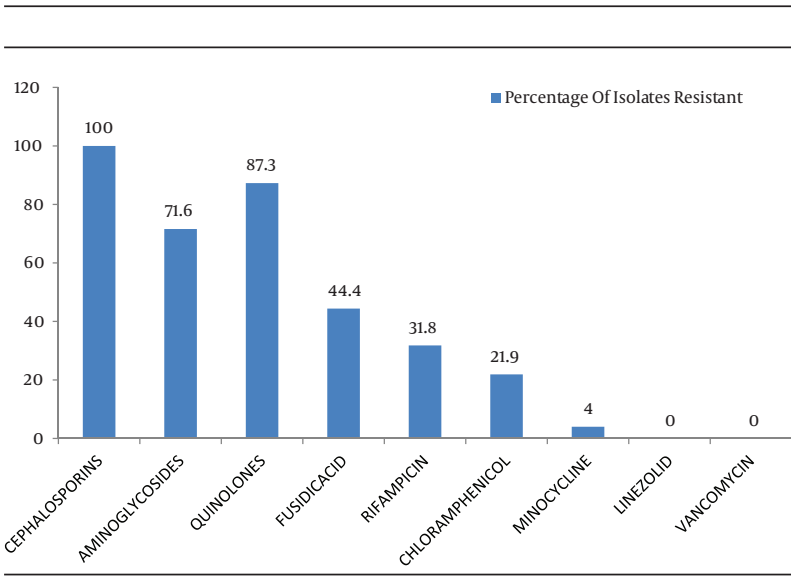

Figure 2. Antibiogram of Methicillin Resistant Coagulase Negative Staphylococci 


\section{Discussion}

Out of 299 isolates, 120 (40.1\%) were found to be methicillin resistant coagulase negative Staphylococci (Figure 1). This amount was substantially higher than the $29.79 \%$ reported by Asma Bashir et al. and 16.2\% by Issam Raad et al. $(8,9)$. This difference may be explained by the different time periods in which the studies were carried out. The continuous and indiscriminate use of antibiotics during this time period resulted in an increase in the proportion of organisms with the resistant genes, hence, giving a higher incidence of resistant species. Unless this problem is managed in time, more and more organisms will become resistant. Such genes may even be transferred to other unrelated pathogens that are still sensitive to our current arsenal of antibiotics. Another study carried out by Issam Raad et al. in Texas University showed that more than $80 \%$ of nosocomial S. epidermidis isolates were methicillin-resistant (6).

The five most effective drugs against the methicillin resistant coagulase negative Staphylococci were vancomycin, linezolid, chloramphenicol, minocycline, and rifampicin. None of the organisms were found to be resistant against linezolid. This is in accordance to a study by Sacar et al. showing that the antibiotic was highly effective in reducing the colony counts in MRSE-infected vascular Dacron grafts in rats which was comparable to the efficacy of vancomycin in the same study (10). Chlorampheniacol was moderately effective with $21.9 \%$ resistance (Figure 2). An earlier study by Fukada et al. showed that this drug had an efficacy rate of $81 \%$ against methicillin resistant $S$. aureus eye infections (2).

It had a relatively better efficacy in such resistant organisms, compared to groups of antibiotics, which allow the administration of this antibiotic for treatment of infections caused by such organisms. Vancomycin was highly effective with $100 \%$ effectiveness, which was greater than 81.2\% reported in previous studies (6). However, it has been reported that the failures in vancomycin treatments are possibly due to the biofilm synthesis, and rifampicin high efficacy, the combination of these antibiotics has been advised (11). This is in accordance with our study in which the mentioned drug was moderately effective against the resistant organisms with $31 \%$ resistance.

This study also pointed out the possibility that vancomycin may be used in combination with linezolid, rifampicin or chloramphenicol, all of the latter drugs have acceptable efficacy against the above mentioned organisms. Linezolid may also be combined to rifampicin for greater efficacy and lower chances of resistance development, as shown by a study the additive effectiveness in a number of cases. Another antibiotic that should be highlighted is minocycline. Its efficacy against the resistant organisms was satisfactory. One study in particular concluded the its high efficiency in eradicating microorganisms embedded in fresh and mature biofilm adhering to catheter surfaces (6). This is important since this drug is much less expensive than both linezolid and vancomycin and has much better efficacy than the other tested antibiotics, that placed it on the top of first line drugs against the resistant organism. Other further studies provided evidences on the possibility of combining minocycline with rifampicin to coat vascular catheters to reduce the probability of infection (12).

Fusidic acid was effective in $55.6 \%$ of cases. This moderate degree of resistance was predicted by a study, reported that it was in-vitro active but the resistance developed if it was used as a single drug treatment and that greater results may be achieved if used in combination with other antibiotics such as rifampicin. This study also concluded that a high level of resistance was found among the organisms against other antibiotics including aminoglycosides and quinolones (Figure 2). Similar results were obtained by Yameen and coworkers for ciprofloxacin and cephalosporins (13). Such high resistivity patterns for the above mentioned drugs could be explained by previous practiced using single drug therapy as well as their excessive use in the hospitals and community resulting in decreased efficacy.

Our study demonstrated that chloramphenicol, vancomycin, linezolid and minocycline all have excellent inhibitory effects against methicillin resistant coagulase negative Staphylococci (Figure 2). These may be used in infections including infective endocarditis, post operation surgical site infections, neonate meningitis and Staphylococcal eye infections, all of which caused by above mentioned organisms as one of the more important causative agents. The oral administration and affordable cost of minocycline, chloramphenicol and linezolid makes these drugs proper to be used for the treatment of such resistant infections. Furthermore, impregnation of medical devices by these agents would decrease the incidence of nosocomial infections which lessen the burden on our health care systems. This study can be further extended to include more samples, newly developed antibiotics and their combinations with other agents allowing us to effectively manage resistant CoNS, in both community and hospital.

\section{Acknowledgements}

The authors hereby acknowledge the great support and guidance of Dr. Afreensih Hassan in designing and writing of the manuscript.

\section{Authors' Contribution}

Muhammad Usman Shah: data collection, literature review and writing of the manuscript, Muhammad Farhan Akram: data collection, literature review and writing of the manuscript, Javaid Usman: selected and presented the basic theme of the article, supervised the study. Fatima Kaleem: selected and presented the basic theme of the article, supervised the study. 


\section{Financial Disclosure}

The authors have no financial interests related to the material in the manuscript.

\section{Funding/Support}

There is no Funding/ Support.

\section{References}

1. Kloos WE, Bannerman TL. Update on clinical significance of coagulase-negative staphylococci. Clin Microbiol Rev. 1994;7(1):117-40.

2. Fukuda Masahiko, Ohashi Hideyuki, Matsumoto Chota, Mishima Soichiro, Shimomura Yoshikazu. Methicillin-Resistant Staphylococcus aureus and Methicillin-Resistant Coagulase-Negative Staphylococcus Ocular Surface Infection. Cornea. 2002;21(Supplement 2):S86-S89.

3. Gill SR, Fouts DE, Archer GL, Mongodin EF, Deboy RT, Ravel J, et al. Insights on evolution of virulence and resistance from the complete genome analysis of an early methicillin-resistant Staphylococcus aureus strain and a biofilm-producing methicillin-resistant Staphylococcus epidermidis strain. J Bacteriol. 2005;187(7):2426-38.

4. Cheung GY, Otto M. Understanding the significance of Staphylococcus epidermidis bacteremia in babies and children. Curr Opin Infect Dis. 2010;23(3):208-16.

5. Favre B, Hugonnet S, Correa L, Sax H, Rohner P, Pittet D. Nosocomial bacteremia: clinical significance of a single blood culture positive for coagulase-negative staphylococci. Infect Control Hosp Epidemiol. 2005;26(8):697-702.

6. Raad I, Chatzinikolaou I, Chaiban G, Hanna H, Hachem R, Dvorak
T, et al. In vitro and ex vivo activities of minocycline and EDTA against microorganisms embedded in biofilm on catheter surfaces. Antimicrob Agents Chemother. 2003;47(11):3580-5.

7. Sharma R, Sharma CL, Kapoor B. Antibacterial resistance: current problems and possible solutions. Indian JMed Sci. 2005;59(3):1209.

8. Bashir A, Mujahid TY, Jehan N. Antibiotic resistance profile: isolation and characterization of clinical isolates of staphylococci from patients with community-acquired skin infections. Pak $J$ Pharm Sci. 2007;20(4):299-304.

9. Raad I, Alrahwan A, Rolston K. Staphylococcus epidermidis: emerging resistance and need for alternative agents. Clin Infect Dis. 1998;26(5):1182-7.

10. Sacar Suzan, Sacar Mustafa, Kaleli Ilknur, Toprak Semra, Cevahir Nural, Teke Zafer, et al. Linezolid compared with vancomycin for the prevention of methicillin-resistant Staphylococcus aureus or Staphylococcus epidermidis vascular graft infection in rats: A randomized, controlled, experimental study. Curr Therap Res. 2007;68(1):23-31.

11. Ford CW, Hamel JC, Wilson DM, Moerman JK, Stapert D, Yancey RJ, Jr, et al. In vivo activities of U-100592 and U-100766, novel oxazolidinone antimicrobial agents, against experimental bacterial infections. Antimicrob Agents Chemother. 1996;40(6):1508-13.

12. Darouiche RO, Raad II, Bodey GP, Musher DM. Antibiotic susceptibility of staphylococcal isolates from patients with vascular catheter-related bacteremia: potential role of the combination of minocycline and rifampin. Int J Antimicrob Agents. 1995;6(1):3136.

13. Yameen Muhammad Arfat, Nasim Hina, Akhtar Naeem, Iram Saira, Javed Imran, Hameed Abdul. Antibiotic susceptibility profile of methicillin-resistant staphylococci isolated from nasal samples of hospitalized patients. Afr J Microbiol Res. 2010;4(3):204-209. 